\title{
Water: The First Archaic Mutagen of Evolution, the Adoptive Mother of Currently Nucleic Acids
}

\section{Fabio Caradonna}

Dipartimento di Scienze e Tecnologie Biologiche Chimiche e Farmaceutiche (STEBICEF, Sezione di Biologia cellulare) Università di Palermo, Viale delle Scienze, Edificio, Palermo, 16 - 90128, Italia

*Corresponding author: Fabio Caradonna, Dipartimento di Scienze e Tecnologie Biologiche Chimiche e Farmaceutiche (STEBICEF, Sezione di Biologia cellulare) Università di Palermo, Viale delle Scienze, Edificio, Palermo, 16 - 90128, Italia, Tel: +39-91-23897331; E-mail: fabio.caradonna@unipa.it

Received date: September 21, 2016; Accepted date: September 22, 2016; Published date: September 27, 2016

Copyright: (c) 2016 Caradonna F. This is an open-access article distributed under the terms of the Creative Commons Attribution License, which permits unrestricted use, distribution, and reproduction in any medium, provided the original author and source are credited.

Keywords: Evolutionary mutagenesis; Evolutionary genetics

\section{Dear Editor}

It would be intuitive to think that for the evolutionary onset of nucleic acids, and even before of their monomeric constituents, the water was crucial.

Within the series of evolutions which start from the origin of the universe and still cannot, by definition, be considered concluded, certainly among the geothermal evolution end and the beginning of the chemical one, on Earth, the moment was favorable for the arrival of the first proto-nucleotides: from underground deposits of methanehydrate [1] and phosphate, with the support of all known pre-biotic physical-chemical conditions, were made the monomeric components of nucleic acids. The cradle of nucleic acids does not seem to have been so fundamentally aqueous, but organic. In fact, thinking back that "all the major biopolymers are metastable in aqueous solution" [2], it is easy to conclude that in those days a proto-nucleotide, or better its carbohydrate, in the aqueous phase would have shown all its inadequacy. However, knowing how today is made a nucleic acid, it is evident that the fine evolutionary strategy has distinguished, for this event, two necessities and consequently has chosen two evolutionary times and two different environments to achieve them:

1. To ensure the synthesis, the events have selected an underground environment basically non-aqueous and consequently not hostile to the nucleotides in general and to the carbohydrate component, in particular. Additionally, the evolutionary randomness system selected ribose as more stable pentose.

2. To guarantee the flexibility, in terms of information variability that the future nucleic acid must keep for not stop the subsequent biological evolution, it was necessary to introduce this new chemical molecule in an environment that can determine a controlled and controllable instability.

In fact, with the advent of the ancestral rains, aqueous surface environment has triggered a sort of proto-mutation. Today, mutagenesis teaches us that in the water can, for example, take place more easily the deamination that converts cytosine into uracil, adenine into hypoxanthine and guanine into xanthine [3], all transformations that change, in a DNA or RNA, the genetic significance of the coded message, adding variability. From this point of view, water could be classified as the first archaic mutagen of evolution. Studies of the $80 \mathrm{~s}$ of the last century also show that the water is able to exchange the chemical-physical parameters of the DNA double helix by moving it from a conformation to another one: for example, the DNA-A is assumed when it is in the presence of little water and instead form B is taken in the presence of a lot of water [4]; this is because the water modifies the strength of hydrogen bonds bridge and the Coulomb forces [5]. Since epigenomics and interactomics reporte today that several biomolecular meanings are associated with different conformations of DNA helix, we can say that the water has actually modulated and continues to modulate the structure and the function of a nucleic acid.

Coming to the current today's genomes we can conclude that the presence of a DNA in an aqueous substrate encourages its instability conformational and informational, helping to give to living organisms the rudimentary genetic variability [6] and ensuring the biosphere for the continuation of biological evolution.

We can define the water as the adoptive mother of nucleic acids: a mother which has not given birth but has accompanied for the rest of existence that which has not generated.

Sincerely

Fabio Caradonna, $\mathrm{PhD}$

\section{References}

1. Ostrovskii VE, Kadyshevich EA (2014) Life Origination Hydrate Theory (LOH-Theory) and the explanation of the biological diversification. J Mol Evol 79: 155-178.

2. Cairns-Smith AG (1982) Genetic takeover and the mineral origins of Life. Cambridge University Press, Cambridge.

3. Neveu M, Kim HJ, Benner SA (2013) The "strong" RNA world hypothesis: fifty years old. Astrobiology 13: 391-403.

4. Saenger W, Hunter WN, Kennard O (1986) DNA conformation is determined by economics in the hydration of phosphate groups. Nature 324: 385-388.

5. Korolev N, Lyubartsev AP, Laaksonen L, Nordensköld L (2002) On the competition between water, sodium ions, and spermine in binding to DNA: a molecular dynamics computer simulation study. J Biophys J 82: $2860-2875$.

6. Catanzaro I, Naselli F, Saverini M, Giacalone A, Montalto G, et al. (2012) Cytochrome P450 2E1 variable number tandem repeat polymorphisms and health risks: a genotype-phenotype study in cancers associated with drinking and/or smoking. Mol Med Rep 6: 416-420. 